\title{
1 Novel Internalin P homologs in Listeria
}

Kayla N Conner ${ }^{1,2, \#}$, Joseph T Burke ${ }^{1,3,4, \#}$, Janani Ravi ${ }^{1,4, *}$, Jonathan W Hardy 1,2,*

${ }^{1}$ Department of Microbiology and Molecular Genetics, Michigan State University, East Lansing, MI, United States.

${ }^{2}$ Institute for Quantitative Health Science and Engineering, Michigan State University, East

Lansing, MI, United States.

${ }^{3}$ Genomics and Molecular Genetics Undergraduate Program, College of Natural Science,

10 Michigan State University, East Lansing, MI, United States.

$11{ }^{4}$ Department of Pathobiology and Diagnostic Investigation, Michigan State University, East

12 Lansing, MI, United States.

13 "Co-primary authors, contributed equally.

14 *Co-Corresponding authors. hardyjon@msu.edu, janani@msu.edu

Keywords: Listeria, Internalin, InIP, Placenta, Molecular evolution, Phylogeny

\section{Impact Statement}

20 The intracellular bacterial pathogen Listeria monocytogenes can breach protective

21 barriers in the pregnant host, allowing for the colonization of the placenta in pregnant

22 women and resulting in numerous adverse pregnancy outcomes. Previous studies aimed

23 at delineating the mechanisms behind placental colonization of $L$. monocytogenes

24 identified a key virulence factor, internalin P (InIP). The internalin family of proteins has

25 been studied extensively due to their conservation in the Listeria genus and their

26 contribution to virulence and pathogenicity in L. monocytogenes. Still, many questions

27 remain regarding the evolution of internalins and their potential roles in non-pathogenic

28 Listeria. Our work addresses this gap in knowledge by 1) identifying additional InIP

29 homologs in Listeria, including L. ivanovii, L. seeligeri, L. innocua, and L. costaricensis,

30 and 2) characterizing these homologs using computational evolutionary methods to

31 compare their primary sequences, domain architectures, and structural models.

32 Together, our findings contribute to the field by providing insights into the evolution of one

33 key member of the internalin family as well as serving as a catalyst for future studies of

34 InIP and its role in Listeria pathogenesis. 


\section{Abstract}

37 Listeria monocytogenes $(L m)$ is a bacterial pathogen that causes listeriosis in 38 immunocompromised individuals, particularly pregnant women. Several virulence factors 39 support the intracellular lifecycle of $L m$ and facilitate cell-to-cell spread, allowing it to 40 occupy multiple niches within the host and cross protective barriers, including the 41 placenta. One family of virulence factors, internalins, contributes to $L m$ pathogenicity by 42 inducing specific uptake and conferring tissue tropism. Over 25 internalins have been 43 identified thus far, but only a few have been extensively studied. Internalins contain

44 leucine-rich repeat (LRR) domains which enable protein-protein interactions, allowing $L m$

45 to bind host proteins. Notably, other Listeria species express internalins but cannot 46 colonize human hosts, prompting questions regarding the evolution of internalins within

47 the genus Listeria. Internalin P (InIP) promotes placental colonization through interaction 48 with the host protein afadin. Though prior studies of InIP have begun to elucidate its role 49 in $L m$ pathogenesis, there remains a lack of information regarding homologs in other

50 Listeria species. Here, we have used a computational evolutionary approach to identify 51 InIP homologs in additional Listeria species. We found that L. ivanovii londoniensis (Liv) 52 and $L$. seeligeri $(L s)$ encode InIP homologs. We also found InIP-like homologs in $L$. 53 innocua and the recently identified species L. costaricensis. All newly identified homologs

54 lack the full-length LRR6 and LRR7 domains found in Lm's InIP. These findings inform on 55 the evolution of one key $L m$ virulence factor, InIP, and serve as a springboard for future 56 evolutionary studies of $L m$ pathogenesis as well as mechanistic studies of Listeria 57 internalins. 


\section{Introduction}

71 Prenatal infection remains a major public health concern. Annually, nearly 13 million

72 infants are born prematurely worldwide, and an estimated $30 \%$ of these preterm births

73 can be attributed to prenatal infection, though the actual number may be higher due to

74 the subclinical nature of many prenatal infections ${ }^{1}$. To better detect and treat these

75 infections to prevent adverse pregnancy outcomes, we must better understand the

76 pathogens that cause them. Listeria monocytogenes is widely used in prenatal infection

77 research due to its well-characterized lifecycle and ease of use in laboratory

78 experiments ${ }^{2,3}$.

79 The Listeria genus comprises 17 species, including the human pathogens $L$.

80 ivanovii ( $L i v)$ and $L$. monocytogenes $(L m)^{4}$. These Gram-positive facultative intracellular

81 bacterial pathogens are the most typical causative agent of listeriosis in humans ${ }^{4,5}$. While

82 relatively rare, listeriosis can result in severe morbidity and mortality in

83 immunocompromised individuals ${ }^{5,6}$. Pregnant women are particularly at risk for listeriosis,

84 as $L m$ can colonize the placenta and cause adverse pregnancy outcomes such as 85 preterm birth, neonatal meningitis, miscarriage, and stillbirth ${ }^{5}$. Lm employs several

86 virulence factors that aid in its invasion of various host niches and the breach of protective

87 host barriers ${ }^{5,6}$. Previous studies have addressed the roles of various $\mathrm{Lm}$ virulence

88 factors, such as ActA, Internalin $A(\operatorname{In} \mid A)$, and Internalin $B(\operatorname{In} \mid B)$ in the context of

89 pregnancy ${ }^{7,8}$. Faralla et al. followed up with two studies focusing on Internalin $P$ (InIP), a

90 key virulence factor for the invasion of the placenta ${ }^{9,10}$.

91 Typically, listeriosis begins with the consumption of contaminated food items. Once

92 in the digestive system, $L m$ uses several virulence factors, including the internalins, to

93 colonize gut epithelial cells and spread throughout the host ${ }^{5,6,11}$. Internalins contribute to

94 this spread by conferring tissue tropism; for example, InIA binds E-cadherin on gut

95 epithelial cells while InIB binds C-Met expressed by hepatocytes ${ }^{11}$. These interactions are

96 enabled by Leucine-Rich Repeat (LRR) domains found in all internalins ${ }^{11,12}$. LRR domains

97 are found in an array of functionally diverse proteins across the domains of life, such as

98 ribonuclease inhibitors in eukaryotes (humans, pigs) and virulence factors in prokaryotes

99 (Yersinia pestis, L. monocytogenes) ${ }^{12}$. Internalins may have as few as four (InIG) or as 100 many as fourteen (InIA) LRR domains ${ }^{5,11}$. Notably, internalins and other virulence factors 
101 are relatively well-conserved across the genus, including species that are considered 102 non-pathogenic to humans, such as $L$. seeligeri and $L$. innocua ${ }^{4,13,14}$. While the details 103 remain unclear, differences in pathogenicity have been attributed to minor genetic 104 variations and differences in the expression of virulence factor genes ${ }^{15}$. The precise roles 105 of the various internalin genes in Listeria and their evolutionary relationships remain 106 critical open questions in Listeria biology.

107 Internalin $P(I n I P)$ is an $L m$ virulence factor known to enhance placental 108 colonization in the pregnant host. This is likely accomplished by enabling $L m$ to 109 transcytose through the basal membrane underlying the syncytiotrophoblast, the 110 protective outer layer of placental cells that serves as a barrier between maternal and 111 fetal blood ${ }^{9}$. Further characterization revealed that InIP encompasses nine LRR domains 112 and binds the human protein afadin, which is a nectin-like protein found in cell-cell 113 junctions and thought to play a significant role in cellular adhesion ${ }^{10}$.

$114 \quad$ Initial InIP studies identified a structural homolog of InIP, Lmo2027, in Lm, but 115 information regarding InIP homologs in other Listeria species has been incomplete ${ }^{9}$. In 116 this study, we used comparative genomics and protein sequence-structure-function 117 analyses to identify InIP homologs in the genomes of $L$. seeligeri $(L s)$, L. ivanovii 118 Iondoniensis (Liv), L. innocua (Lin), and L. costaricensis (Lc) (Fig. 3, Table S1). The 119 bioinformatic analysis presented here serves as a springboard for future studies of 120 Listeria evolution and pathogenesis pertaining to the internalin protein family, including 121 its ability to colonize the human placenta.

\section{Materials and Methods}

124 Identification of InIP Homologs. To identify InIP $L m$ homologs across evolutionary 125 lineages, we submitted the InIP $L m$ amino acid sequence (accession: WP_014601135.1) 126 to MolEvolvR (http://www.jravilab.org/molevolvr) ${ }^{16}$. The query returned hits for 127 homologous proteins across bacterial phyla. While many species carried homologous 128 proteins (e.g., Nostoc spp. and Beggiatoa leptomitoformis), we chose to filter out hits with 129 low similarity and divergent domain architectures and genomic contexts for our detailed 130 study; we thus focused on the Listeria genus (including 45,530 L. monocytogenes, 740 131 L. innocua, 169 L. seeligeri, 44 L. ivanovii, and 1 L. costaricensis genomes). Within this 
132 dataset, we selected the hits with the highest percent similarity and unique domain 133 architectures as representative homologs for further analysis (Figure 1; Table S1).

134 Accession numbers provided by MolEvolvR were used to query the NCBI RefSeq Protein

135 Database for corresponding nucleotide sequences and locus tags for homologous 136 genes ${ }^{17}$. BioCyc ${ }^{18}$ (https://biocyc.org) and NCBI RefSeq ${ }^{17}$ protein databases were used 137 to identify genomic contexts (neighboring genes).

139 Calculation of Percent Identity and Percent Similarity. Percent identity and percent 140 similarity values for predicted homologs were provided by MolEvolvR ${ }^{16}$ (Table S1; 141 https://github.com/iravilab/inlp listeria). Nucleotide sequences for inlP in $L$. 142 monocytogenes, L. ivanovii londoniensis, L. seeligeri, and L. costaricensis were aligned 143 using Clustal Omega $^{19}$ (https://www.ebi.ac.uk/Tools/msa/clustalo/), and resulting 144 alignments were submitted in FASTA format to the Sequence Manipulation Suite ${ }^{20}$ (SMS; 145 http://www.bioinformatics.org/sms2) to calculate percent nucleotide identity. The homolog 146 similarity and identity matrix was generated using MatGAT2.01 with the BLOSUM 62 147 matrix and default options ${ }^{21}$.

149 Multiple Sequence Alignment, Phylogenetic Trees, and Protein Models. Multiple 150 sequence alignments for homologous amino acid sequences were generated using 151 Kalign $^{24}$ and visualized using JalView (Version 2.11.1.4) ${ }^{25}$. Phylogenetic trees were 152 constructed using Kalign ${ }^{24}$, FastTree, and FigTree. Three-dimensional protein models 153 were produced using SWISS-MODEL with the $L$. monocytogenes Internalin $\mathrm{P}$ crystal 154 structure (PDB: $5 \mathrm{hl} 3$ ) as a template ${ }^{26,27}$. All data, analyses, and visualizations for InIP 155 Listeria homologs are available here: https://github.com/jravilab/inlp listeria. 


\section{Results}

\section{Listeria ivanovii londoniensis and Listeria seeligeri encode Internalin P Homologs.}

164 To begin investigating evolutionary conservation of the L. monocytogenes Internalin P 165 (InIP $L m)$, we started with an extensive homology search and protein characterization of 166 InIP-like proteins in diverse lineages across the tree of life using MolEvolvR ${ }^{16}$ 167 (http://iravilab.org/molevolvr). Most homologs were present only within the genus Listeria. 168 To further ensure that all homologs are being identified, we picked other representative 169 InIP homologs from L. ivanovii and L. seeligeri as new starting points for our homology 170 search and characterization (using MolEvolvR ${ }^{16}$; see Methods). We found several hits in 171 our multi-start search including proteins that resembled other Internalins (e.g., InIB), and 172 others that carried neither the signature Internalin_N or LRR domains characteristic of 173 internalins (Fig. 2 and S1). Therefore, we restricted our full set of homologs to only InIP174 like proteins resulting in 64 representative proteins with distinct domain architectures from 175 each Listeria species including L. monocytogenes, L. seeligeri, L. ivanovii, L. innocua, 176 and L. costaricensis (Fig. 2 and S1; Table S1). Homologs from L. seeligeri ( $L s)$ and $L$. 177 ivanovii (Liv) showed $>65 \%$ amino acid similarity compared to InIP $L m$, while homologs 178 from L. innocua ( $L i n)$ and L. costaricensis ( $L C$ ) showed $<35 \%$ and $55 \%$ similarity, 179 respectively (Fig. 3). We found that several homologs lacked predicted signal peptide 180 domains suggesting they are not secreted like InIP; this was further corroborated by the 181 presence of predicted transmembrane LPXTG motifs, which indicate that these homologs 182 are more likely to be membrane-anchored InIB-like proteins rather than secreted InIP 183 homologs (Fig. 2 and S1). Investigation of the InIP-like proteins in LC and Lin showed 184 that it is unlikely that they are functional InIP homologs (discussed below). Further 185 investigation of domain architectures and genomic contexts of putative homologs 186 ultimately revealed one InIP homolog encoded within the L. ivanovii londoniensis genome 187 and three InIP homologs encoded by L. seeligeri (discussed below). Here, we refer to 188 these homologs as $\operatorname{InI} P_{L m}$, InIP $P_{L i}, \operatorname{InI} \mid P_{L s 1}, \operatorname{InI} P_{L s 2}$, and $\operatorname{InI} P_{L s 3}$, respectively, to indicate 189 species and gene order.

190 We determined the similarity of InIP homologs in Liv and $L s$ at the nucleotide and 191 amino acid levels towards functional characterization. The inIP gene in L. ivanovii 192 Iondoniensis $\left(i n / P_{L i v}\right)$, as well as the three L. seeligeri paralogs $\left(i n I P_{L s 1}\right.$, inIPLs2, and 
193 inIP $\left.P_{L s 3}\right)$, shared $\sim 70 \%$ identity with the inI $P_{L m}$ gene and $\sim 52-65 \%$ identity at the amino

194 acid level when compared to InIP $L m$ (Fig. S2). However, the newly identified homologs in

195 L. ivanovii londoniensis and L. seeligeri shared much higher percent amino acid similarity 196 with $\operatorname{InI} \mathrm{P}_{L m}$ - InIP $\mathrm{P}_{L i v}$, InIP $\mathrm{P}_{L s 1}$, and InIP $\mathrm{P}_{L s 3}$ showed $70 \%$ similarity to $\operatorname{InI} \mathrm{P}_{L m}$ (Fig. 3). InIP $\mathrm{P}_{L s 2}$

197 showed a striking 94\% similarity to the L. monocytogenes InIP (Fig. S2). Notably, the 198 flanking $L$. seeligeri paralogs, $I_{n I P} P_{L s 1}$ and $I_{L I} P_{L s 3}$, were more similar to each other than to 199 the third paralog or to $\operatorname{InIP}_{L m}$ (Fig. 3). To further investigate these new Listeria InIP 200 proteins, we next explored their genomic neighborhoods.

Because the Listeria genus maintains a high degree of synteny across species,

202 we investigated the genomic contexts of identified homologs compared to the InIP $L m$ 203 gene, which is flanked upstream by an amino acid permease gene and downstream by 204 an NADPH dehydrogenase gene (Fig. 4A). We hypothesized that functional homologs of 205 InIP $L m$ would be flanked by these same genes in other Listeria species. We, therefore, 206 determined the genomic neighborhoods of inIP homologs in L. ivanovii londoniensis, $L$. ivanovii ivanovii, L. seeligeri, L. costaricensis, and L. innocua (see Methods). We found 208 that the inIP genes in L. ivanovii londoniensis and L. seeligeri were flanked upstream by 209 an amino acid permease gene and downstream by an NADPH dehydrogenase gene 210 mirroring the L. monocytogenes genomic context, suggesting the identified homologs are 211 likely true homologs of the inIP gene (Fig. 4). Interestingly, we found that the gene 212 encoding the InIP-like protein in Lc was flanked upstream by an amino acid permease 213 and downstream by a gene encoding a LapB repeat-containing protein, inconsistent with 214 genomic neighborhoods seen in other Listeria species (Fig. 4E). Also inconsistent with 215 other genomic neighborhoods, the InIP-like protein in Lin was flanked upstream by a 216 DUF5110-containing protein-encoding gene and downstream by the ssrA gene (Fig. 4F).

217 To quantify the similarity of the flanking genes in L. ivanovii londoniensis, L. seeligeri, and 218 L. monocytogenes, we calculated their pairwise similarity. At the amino acid level, the 219 products of these flanking genes had $>95 \%$ similarity to L. monocytogenes (Fig. S3). 220 Notably, while L. ivanovii londoniensis encoded an inIP homolog in this region, L. ivanovii 221 ivanovii did not (Fig. 4). Additionally, while L. ivanovii londoniensis encoded only one copy 222 of inIP (EL212_RS12905; inIPLi), L. seeligeri encoded three copies (LSE_RS12040, LSE_RS12045, and LSE_RS12050; inIPLs1, inIPLs2, and inIPLs3, respectively) (Fig. 4). 
224 Internalin P Homologs in L. ivanovii and L. seeligeri lack the full-length LRR6 and 225 LRR7 domains found in L. monocytogenes InIP.

226 To delineate the evolution of InIP within Listeria, we generated a multiple sequence 227 alignment (Fig. 5) and constructed a phylogenetic tree of the homologs (Fig. 1). While 228 we observed several amino acid substitutions throughout the length of the proteins, the 229 most striking difference between InIP $L m$ and its homologs was in the Leucine-Rich Repeat 230 (LRR) regions - a partial lack of LRR6 and complete lack of LRR7 - in the L. ivanovii 231 and L. seeligeri homologs (Fig. 1). Additionally, we noted a lack of conservation in a

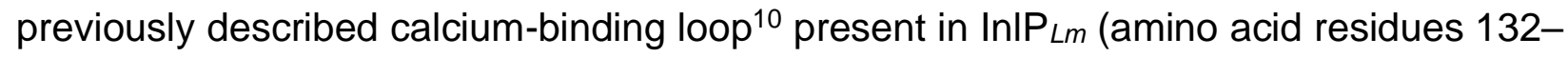
233 135; Fig. 1). This observation was of particular interest since this calcium-binding loop 234 might play a role in protein signaling or stabilization of protein-protein interactions 235 between InIP $L m$ and host afadin. To better visualize the structural differences in these 236 homologs, we generated models of $\operatorname{In|}\left|P_{L i}, \operatorname{In}\right| P_{L s 1}, \operatorname{In} \mid P_{L s 2}$, and $\operatorname{In|} \mid P_{L s 3}$ based on the 237 previously resolved crystal structure of $I n I P_{L m}$ (Fig. 1; see Methods). These models 238 illustrate the similarity in the overall structure of the five homologous proteins, and the 239 lack of LRR7 and full-length LRR6 are visible in Li and Ls homologs (Fig. 1; green/yellow 240 regions). Additionally, the calcium-binding loop region is discernible in all five homologous 241 proteins but appears structurally diverse in L. ivanovii and L. seeligeri homologs.

242 In summary, we have discovered novel Internalin P homologs in Listeria, traced 243 their evolution, and uncovered potential functional implications pertaining to 244 heterogeneity in key InIP domains. All InIP homolog data (along with characterizations in 245 terms of domain architectures and modeling) are available at 246 https://github.com/iravilab/inlp listeria. 


\section{Discussion}

255 While previous studies have addressed many of the physical and mechanistic 256 properties of InIP, the conservation of the inIP gene within or outside of the Listeria genus 257 remains incompletely characterized. Here, we have provided insight into Internalin $P$ in 258 other Listeria species aside from L. monocytogenes that will drive future mechanistic 259 studies of InIP as well as evolutionary studies of Listeria pathogenesis pertaining to the 260 internalins.

261 First, we analyzed the InIP amino acid sequence with MolEvolvR ${ }^{16}$ to retrieve 262 homologous proteins across evolutionary lineages. MolEvolvR ${ }^{16}$ is a powerful new 263 bioinformatic web application to characterize protein families using molecular evolution 264 and phylogeny (http://jravilab.org/molevolvr). The MolEvolvR ${ }^{16}$ InIP search returned a list 265 of potential homologs including those found in Listeria species. In this article, we focus 266 on homologs in Listeria since these species carried the classic Internalin and LRR 267 domains. Using MolEvolvR ${ }^{16}$, we identified InIP-like proteins in L. innocua, L. seeligeri, $L$. 268 ivanovii, and L. costaricensis; only homologs in L. seeligeri and L. ivanovii londoniensis 269 expressed an amino acid percent similarity value $>65 \%$. We found that the lower identity 270 proteins are more likely to be Internalin B homologs based on their sequence, domain 271 architecture, and structure.

272 To determine if the newly identified proteins were true homologs of $\operatorname{InIP} L m$, we 273 explored their domain architectures and genomic context. Consistent with the synteny 274 observed in Listeria genomes, we found that the inIP domain architectures and genomic 275 neighborhoods were highly conserved in L. ivanovii londoniensis and L. seeligeri, but not 276 in L. ivanovii ivanovii, L. innocua, or L. costaricensis. While it is possible that these species 277 could encode inlP homologs elsewhere in their genomes, it seems unlikely considering 278 their domain architectures and lower conservation in sequence compared to other 279 homologs. It is more likely that the homologous proteins identified in $L C$ and $L$ in are 280 independent of InIP, but in the same class of small, secreted internalins that 281 encompasses InIP, InIC, and InIH, among others ${ }^{11}$.

282 Notably, we found that $L$. ivanovii londoniensis encoded a homolog for inlP while 283 L. ivanovii ivanovii did not. Historically, L. ivanovii londoniensis and L. ivanovii ivanovii 284 have been distinguished biochemically ${ }^{28}$. Recently, Hupfeld et al., noted that the two 
285 subspecies could also be distinguished based on bacteriophage susceptibility: L. ivanovii 286 ivanovii strains are sensitive to bacteriophages, while L. ivanovii londoniensis strains 287 encode a type II-A CRISPR-Cas system rendering them resistant to many phages ${ }^{29}$. Our 288 finding that only $L$. ivanovii londoniensis, and not $L$. ivanovii ivanovii, encodes the inIP 289 gene provides another avenue for distinguishing between these two subspecies and 290 could be beneficial to public health laboratories seeking to differentiate between them 291 among clinical and food isolates. Additionally, because the evolution of virulence factors 292 in Listeria remains mysterious, the specific presence of inlP in subspecies such as 293 ivanovii londoniensis and its absence in ivanovii ivanovii may provide clues as to how 294 Listeria evolves the ability to infect different cells and tissues.

Since L. ivanovii has been implicated in human and animal placental infection, it was not entirely surprising to find that it encoded the gene for InIP, an internalin known to 297 enhance placental colonization. It was surprising, however, to find three copies of the inIP 298 gene in L. seeligeri since it has not been significantly indicated in human or animal 299 pathogenesis ${ }^{30}$. Our analyses suggested that $\operatorname{InIP} L s 2$ was the most similar to $\operatorname{InIP} L m$ and $300 \quad \operatorname{InI} P_{L i v}$. It is possible that this copy $\left(\operatorname{InI} P_{L s 2}\right)$ is the ancestral one, and $\operatorname{InI} P_{L s 1}$ and $\ln \mid P_{L s 3}$ 301 resulted from subsequent duplication events. The presence of multiple homologs 302 suggests that InIP could have alternative functions apart from enhancing placental 303 colonization. Listeria species are frequently found in environmental isolates, as they 304 readily reside in soil. It is possible that InIP provides a fitness advantage in this 305 environment.

$306 \quad$ One of the main questions resulting from the discovery of InIP homologs centers 307 on the evolutionary timeline of the Listeria genus: which InIP came first? Our discovery 308 that InIP $\mathrm{P}_{L i v}, \operatorname{InI} \mathrm{P}_{L s 1}, \operatorname{InI} \mathrm{P}_{L s 2}$, and InIP $\mathrm{Lss}_{3}$ do not contain the full-length LRR6 and LRR7 309 domains found in InIP $\mathrm{Lm}_{\mathrm{m}}$ begins to offer potential answers to this question. It is plausible 310 that L. monocytogenes, L. seeligeri, and L. ivanovii londoniensis shared a common 311 ancestor that passed down the inlP gene, and a subsequent insertion event in $L$. 312 monocytogenes led to the full-length InIP containing LRR6 and LRR7. Conversely, it is 313 likely that L. monocytogenes carries the ancestral copy of InIP (InIP $L m)$; the full-length inIP 314 gene could have undergone a deletion resulting in the loss of LRR6 and LRR7 in InIP Liv 315 and InIP $L s$, although it is less likely to observe several deletion events as against a single 
316 insertion event. Future studies on the evolution of the Listeria genus and the larger family

317 of internalin proteins will be required to answer this question more rigorously and 318 determine their possible links to pathogenicity.

319 An additional structural difference noted between newly identified InIP homologs 320 resides in the $\mathrm{Ca}^{2+}$-binding loop of LRR3. Previously, this loop has been hypothesized to 321 play a role in InIP signaling, activation, or stabilization in complex with its binding partner $322 \operatorname{afadin}^{10}$. Structural heterogeneity is visible in the $\mathrm{Ca}^{2+}$ regions of InIP homolog models; 323 InIP homologs in Liv and $L s$ appear to have more open loops compared to $\operatorname{lnIP} L m$. The 324 ability of these loops to bind calcium, and their relative binding affinities will be an 325 important avenue for future investigation, especially as more details regarding the 326 function and regulation of InIP ${ }_{L m}$ come to light.

Recent studies made several fundamental discoveries regarding the physical and 328 mechanistic properties of $\operatorname{InIP} L m$ and its activity in the placenta, but many questions 329 remain unanswered ${ }^{9,10}$. The discovery of InIP homologs in $L$. ivanovii londoniensis and $L$. 330 seeligeri, two species that have not been substantially implicated in cases of placental 331 infection, is compelling. Future studies will investigate the activity of these homologs to 332 determine if they bind afadin and if they are able to enhance placental colonization of $L$. 333 monocytogenes as well as endogenous InIP. Further, structural differences between 334 these homologs suggest potential binding sites for the InIP-afadin interaction, which has 335 not been resolved to date.

In summary, we report that L. ivanovii londoniensis and L. seeligeri encode 337 homologs for the L. monocytogenes virulence factor InIP. Identified homologs in all three 338 species are housed within similar genomic neighborhoods, flanked by the same 339 housekeeping genes upstream and downstream; further, L. seeligeri encodes three 340 copies of the inIP gene in this region. All four homologs are similar $(>70 \%)$ to InIP in $L$. 341 monocytogenes, the main structural difference resulting from the lack of full-length LRR6 342 and $L R R 7$ regions in $I n \mid P_{L i}, \operatorname{InIP}{ }_{L s 1}, \operatorname{InI} P_{L s 2}$, and $I n \mid P_{L s 3}$. Our findings will serve as a 343 springboard for future evolutionary studies of internalins in the Listeria genus and will 344 bolster future in vitro and in vivo studies of InIP in the context of virulence and 345 pathogenicity. 


\section{Funding}

349 KNC is supported by Michigan State University (MSU) Microbiology and Molecular

350 Genetics departmental fellowships. This work was supported by MSU start-up funds

351 granted to $\mathrm{JWH}$ and $\mathrm{JR}$.

\section{Author contributions}

354 Conceptualization: KNC, JTB, JR, JWH. Methodology: KNC, JTB, JR. Software: JTB,

355 JR. Validation: KNC, JTB, JR, JWH. Formal Analysis: KNC, JTB, JR. Investigation:

356 KNC, JTB, JR. Resources: JR, JWH. Data Curation: KNC, JTB, JR. Writing: KNC, JR,

357 JWH. Visualization: KNC, JTB, JR. Supervision: JR, JWH. Project Administration: JR,

358 JWH. Funding: JR, JWH.

359

360 Data availability and reuse

361 All the data, analyses, and visualizations are available in our GitHub repository, 362 https://github.com/JRaviLab/inlp_listeria. Text, figures, and data are licensed under 363 Creative Commons Attribution CC BY 4.0.

364

\section{Conflicts of Interest}

366 The authors declare that there are no conflicts of interest. 


\section{Figures}

\section{Figure 1: Phylogeny and Structure Models of InIP and Representative Homologs. A}

378 phylogenetic tree was generated using the amino acid sequences of InIP homologs in $L$.

379 monocytogenes (blue), L. ivanovii londoniensis (Purple), and L. seeligeri (green). Three-

380 dimensional models were generated using SWISS-MODEL with the crystal structure for

381 InIP $L m$ (PDB: $5 \mathrm{hl} 3$ ) as a template. Multiple sequence alignments were generated using

382 MolEvolvR and illustrate the complete LRR6 and LRR7 insertion present in InIP Lm. The

383 legend shows the colors of the amino acid residues indicated in the multiple sequence

384 alignment

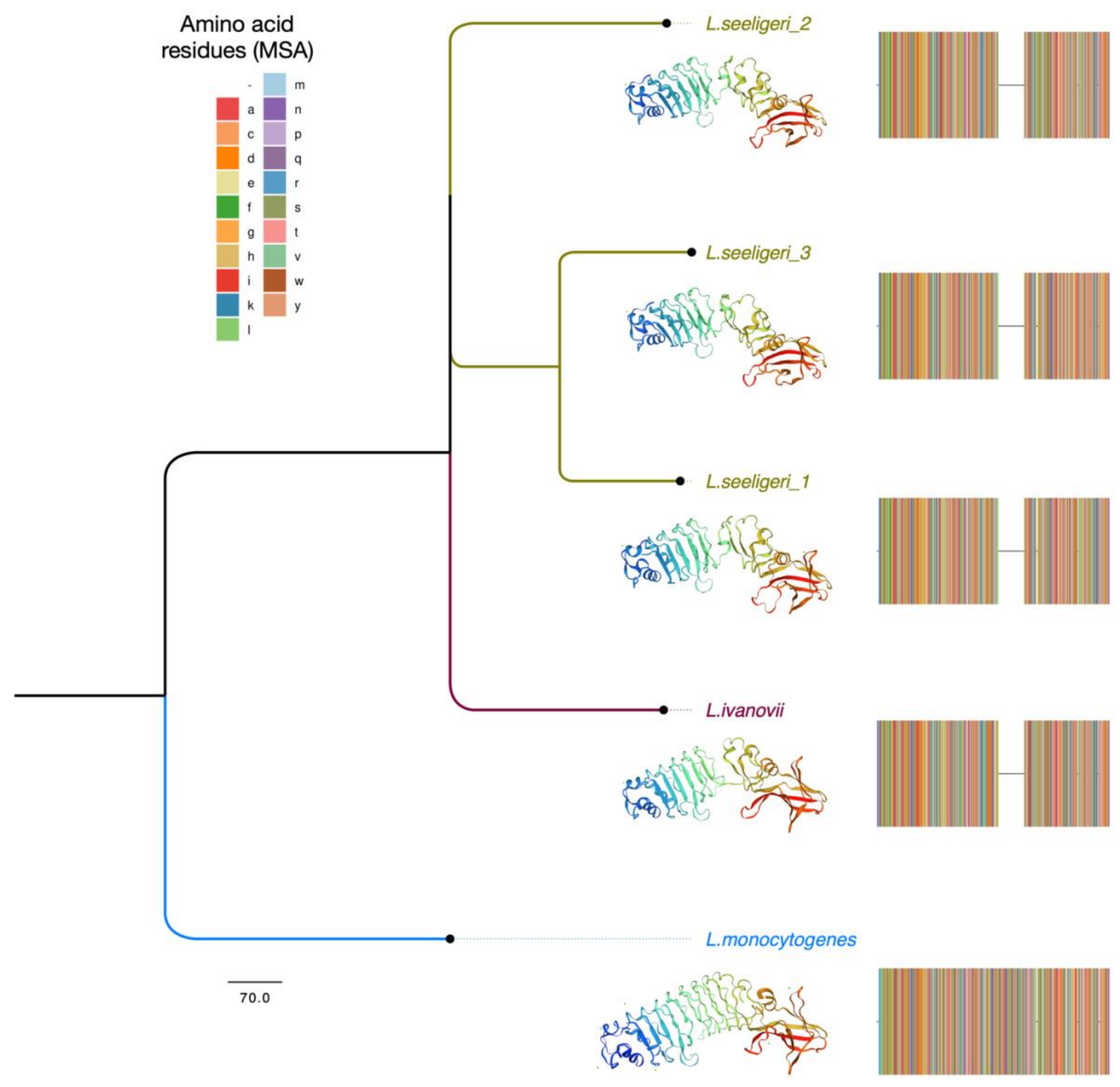


bioRxiv preprint doi: https://doi.org/10.1101/2022.01.19.476994; this version posted January 20, 2022. The copyright holder for this preprint (which was not certified by peer review) is the author/funder, who has granted bioRxiv a license to display the preprint in perpetuity. It is made available under aCC-BY-NC-ND 4.0 International license.

386 Figure 2: Phylogeny and Domain Architectures of Putative Internalin P Homologs.

387 A multiple sequence alignment and phylogenetic tree were generated using the amino

388 acid sequences of putative InIP homologs identified using MolEvolvR and five Listeria

389 InIP starting points (See Methods). The phylogeny of InIP-like proteins has been overlaid

390 with their domain architectures (generated using Pfam database, MolEvolvR). The legend

391 shows the colors of the amino acids indicated in the multiple sequence alignment and

392 Pfam domain annotations.

\section{Domain architecture (Pfam)}

BFirmic_Lmonocytogenes_WP_010990000.1 BFirmic_Lmonocytogenes_WP_096931854.1

BFirmic_Lmonocytogenes_WP $014601135.1-$ D D D

BFirmic_LmonocylogentwP_110115325.1 -

BFirmic_Lmonocytogenes_WP_079442373.1 D D

BFirmic Lmonocytogenes WP_110135527.1 D D

BFirmic_Lmonocytogenes_WP 159107855.1 DP

BFirmic_Lmonocytogenes_WP_140442524.1 $\longrightarrow$

BFirmic_Lseeligeri_WP_046326327.1 $\square \square$

BFirmic_Lseeligeri_WP_012986389.1 -

BFirmic Livanovii WP 111143678.1 -

BFirmic_Lmonocytogenes_WP_149059774.1 -

BFirmic_Lcostaricensis_WP_099224870.1 D D

BFirmic L WP 160464771.1 D

BFirmic Linnocua WP 003769168.1

BFirmic_Lseeligeri_WP_075702544.1 -

BFirmic Lmonocytogenes WP 074467472.1 D

(1) 069027490.1 -

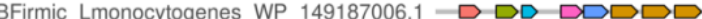

BFirmic_Lmonocytogenes_WP_070034320.1 — D -

BFirmic_Lmonocytogenes_WP_069888097.1 — •

BFirmic_Livanovii_WP_112107399.1 - D

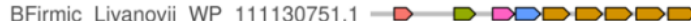

BFirmic_Livanovii_WP_143211001.1 — D D

BFirmic_Livanovii_WP_111151248.1 \ D $\square \square \square$

BFirmic_Livanovii_WP_014091885.1 — • •

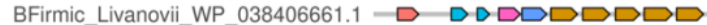

BFirmic_Livanovii_WP_167468914.1 - D D D

BFirmic_Lmonocytogenes_WP_149057981.1 ๑D $\longrightarrow \square \rightarrow \square \square \square \square$

BFirmic_Lmonocytogenes_WP_070222498.1 D D $\longrightarrow$ D

BFirmic Lmonocytogenes WP $070242630.1 \rightarrow$ D D D D D D D

BFirmic_Lmonocytogenes_WP_149041317.1 — DDDD - D

BFirmic_Lmonocytogenes_WP_110164984.1 D DDD DDD

BFirmic_Lmonocytogenes_WP_070241341.1 • DD - $\square$

BFirmic Lmonocytogenes WP 110109784.1 - D DD - D

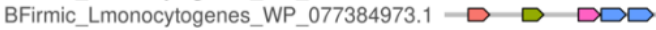

BFirmic_Lmonocytogenes_WP_120186602.1 D DDD DD

BFirmic_Lmonocytogenes_WP_061105368.1 - D D D

BFirmic Lmonocytogenes WP 120244111.1 - D D D

BFirmic Lmonocytogenes_WP_110115303.1 - D D DD

BFirmic_Lmonocytogenes_WP_110097511.1 - D D DD

BFirmic_Lmonocytogenes_WP_110097869.1 - $\quad$ DD D DD

BFirmic Lmonocytogenes WP 061105794.1 - D D D D

BFirmic Lmonocytogenes WP 110115313.1 - D D D D

BFirmic_Lmonocytogenes_WP_003728061.1 D D D D D

BFirmic Lmonocytogenes WP 070295358.1 - D D D

BFirmic Lmonocytogenes_WP $070241340.1=0$

BFirmic Linnocua WP 033533514.1 -

BFirmic_Linnocua_WP_031649074.1 BFirmic L WP 160464919.1 .

BFirmic Lmonocytogenes WP 069000955.1

BFirmic_Lmonocytogenes_WP_149051722.1

BFirmic_Lmonocytogenes_WP_160121597.1

BFirmic_Lmonocytogenes_WP_109908670.1

BFirmic Lmonocytogenes WP 070213144.1 BFirmic_Lseeligeri_WP_075702529.1 BFirmic_Livanovii_WP_111130740.1 BFirmic_Livanovii_WP_112107397.1 BFirmic Livanovii WP_102037871.1 BFirmic Livanovii WP 052010532.1 BFirmic_Linnocua_WP_003765406.1

Representative homologs

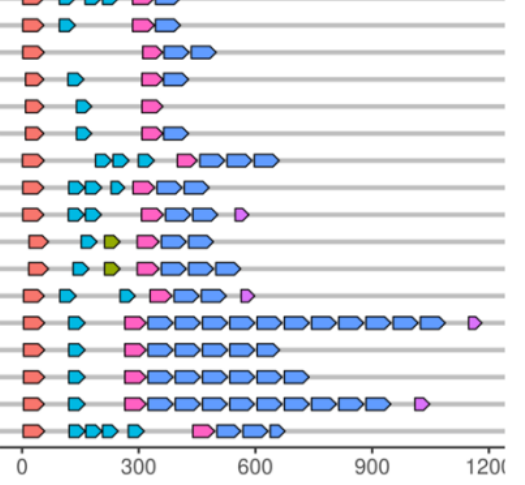

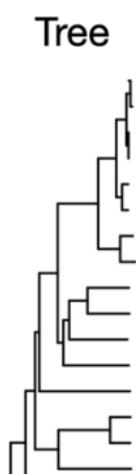
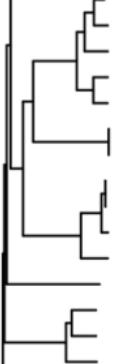

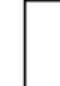

MSA

HнHнH-

HHH

HHHH

패НHI-

HHHI-

패HНH

- IHI

HII

Hin

HHH

HHII

irint

Iㅐ는

HH

$\mathrm{HH}$

If- 개개

H- HНH

Hirifil

H-1

H-1H

H-DH HIH4

내내패

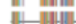

프내패패

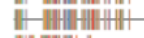

HHAl中

배팬-1

HHirin-

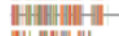

IHHHHII

IHHHIII

H+H

IHIIII

HH

패패개

HWH

패HㅐH

패패패패

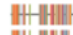

HHH

패핀-1

배패패

IHHIIII

筷HHH

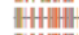

HHHH

HHHH

HHill

HHAH

HHHHH

\#HHH H

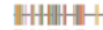

H+HH

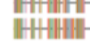

H+hili

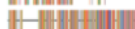

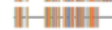

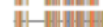

粗-

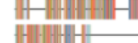

\section{Domain annotations (Pfam)}

Bacterial adhesion/invasion protein $\mathrm{N}$ terminal GW (Gly-Tryp) dipeptide domain

Leucine rich repeat

Leucine Rich repeat

Leucine Rich Repeat

Leucine Rich repeats (2 copies)

Listeria-Bacteroides repeat domain (List_Bact_rpt)

LPXTG cell wall anchor motif

LRR adjacent

Amino acid residues (MSA)

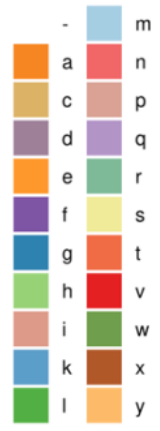


393 Figure 3: Percent Similarity and Identity of Internalin P Homologs in representative

394 Listeria species. Percent similarity and percent identity were calculated for the amino

395 acid sequences for representative Internalin $P$ homologs from five Listeria species, $L$.

396 monocytogenes, L. ivanovii, L. seeligeri, L. costaricensis, and L. innocua. Matrix showing

397 similarity and identity values was generated using MatGAT2.01 with the BLOSUM 62

398 matrix and default options selected.

\begin{tabular}{|c|c|c|c|c|c|c|c|}
\hline Representative InIP homologs & Liv & Lm & Ls1 & Ls2 & Ls3 & Lin & Lc \\
\hline BFirmic_Livanovii_WP_126300113.1 & & 58.1 & 74.6 & 78.6 & 75.2 & 37.8 & 32.1 \\
\hline BFirmic_Lmonocytogenes_WP_014601135.1 & 70.9 & & 57.9 & 57.5 & 57.2 & 32.4 & 38.2 \\
\hline BFirmic_Lseeligeri_WP_012986389.1 & 85.7 & 72.7 & & 77.3 & 90.5 & 36.9 & 33.1 \\
\hline BFirmic_Lseeligeri_WP_012986390.1 & 89.7 & 70.9 & 87.0 & & 76.7 & 36.5 & 30.8 \\
\hline BFirmic_Lseeligeri_WP_012986391.1 & 84.9 & 72.2 & 94.8 & 85.3 & & 36.6 & 32.5 \\
\hline BFirmic_Linnocua_WP_003769168.1 & 61.7 & 52.6 & 58.9 & 60.3 & 58.6 & & 28.1 \\
\hline BFirmic_Lcostaricensis_WP_099224870.1 & 45.7 & 53.0 & 46.1 & 43.4 & 45.9 & 43.0 & \\
\hline
\end{tabular}


416 Figure 4: Genomic Context of Newly Identified Internalin P Gene Homologs. Genes

417 homologous to the $L$. monocytogenes inIP $(\mathrm{A})$ were identified in various other Listeria 418 species. Gene order was maintained in L. monocytogenes, L. seeligeri, and L. ivanovii 419 Iondoniensis. Notably, L. seeligeri encodes three copies of the inIP gene. All homologs 420 categorized as "true" homologs were flanked upstream by an amino acid permease gene 421 (blue) and downstream by an NADPH dehydrogenase gene (orange). L. ivanovii ivanovii 422 contains a pseudogene (purple) and an uncharacterized gene encoding a hypothetical 423 protein (red) in this region. Putative homologous genes in L. costaricensis and L. innocua 424 did not mirror genomic neighborhoods seen in the other Listeria species. All inlP 425 homologs are represented in green. Genomic context was determined using RefSeq 426 genomic records and the BioCyc genome browsers for each species (see Methods).

A. Listeria monocytogenes Genomic Context

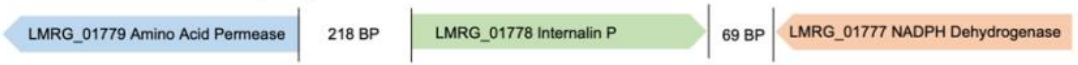

B. Listeria ivanovii londoniensis Genomic Context

\begin{tabular}{l|l|l|l|l} 
EL212_RS12900 Amino Acid Permease & 251 BP & EL212_RS12905 Intermalin P & 96 BP & EL212_RS12910 NADPH Dehydrogenase
\end{tabular}

C. Listeria seeligeri Genomic Context

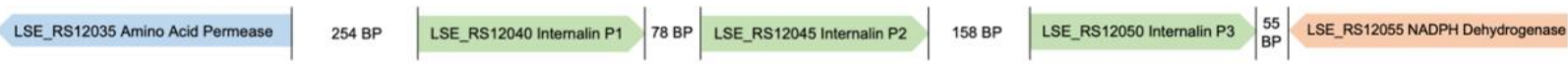

D. Listeria ivanovii ivanovii Genomic Context

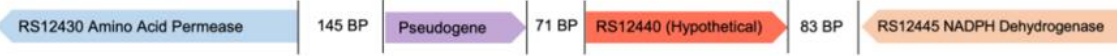

E. Listeria costaricensis Genomic Context

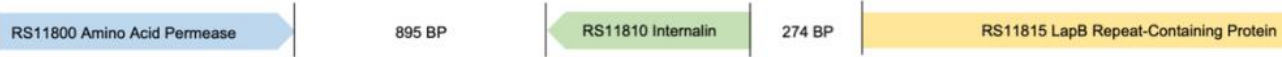

F. Listeria innocua Genomic Context 
bioRxiv preprint doi: https://doi.org/10.1101/2022.01.19.476994; this version posted January 20, 2022. The copyright holder for this preprint (which was not certified by peer review) is the author/funder, who has granted bioRxiv a license to display the preprint in perpetuity. It is made available under aCC-BY-NC-ND 4.0 International license.

434 Figure 5: Multiple Sequence Alignment of Internalin P Homologs. Amino acid 435 sequences for identified Internalin $\mathrm{P}$ homologs in L. monocytogenes, L. ivanovii

436 Iondoniensis, and L. seeligeri were aligned using Kalign and visualized using Jalview.

437 Below the alignment is the consensus sequence for the four homologs. The red box

438 indicates the insertion present only in the InIP $L m$ homolog. The $L$. monocytogenes

439 homolog is used as the reference InIP protein (for residue numbering).

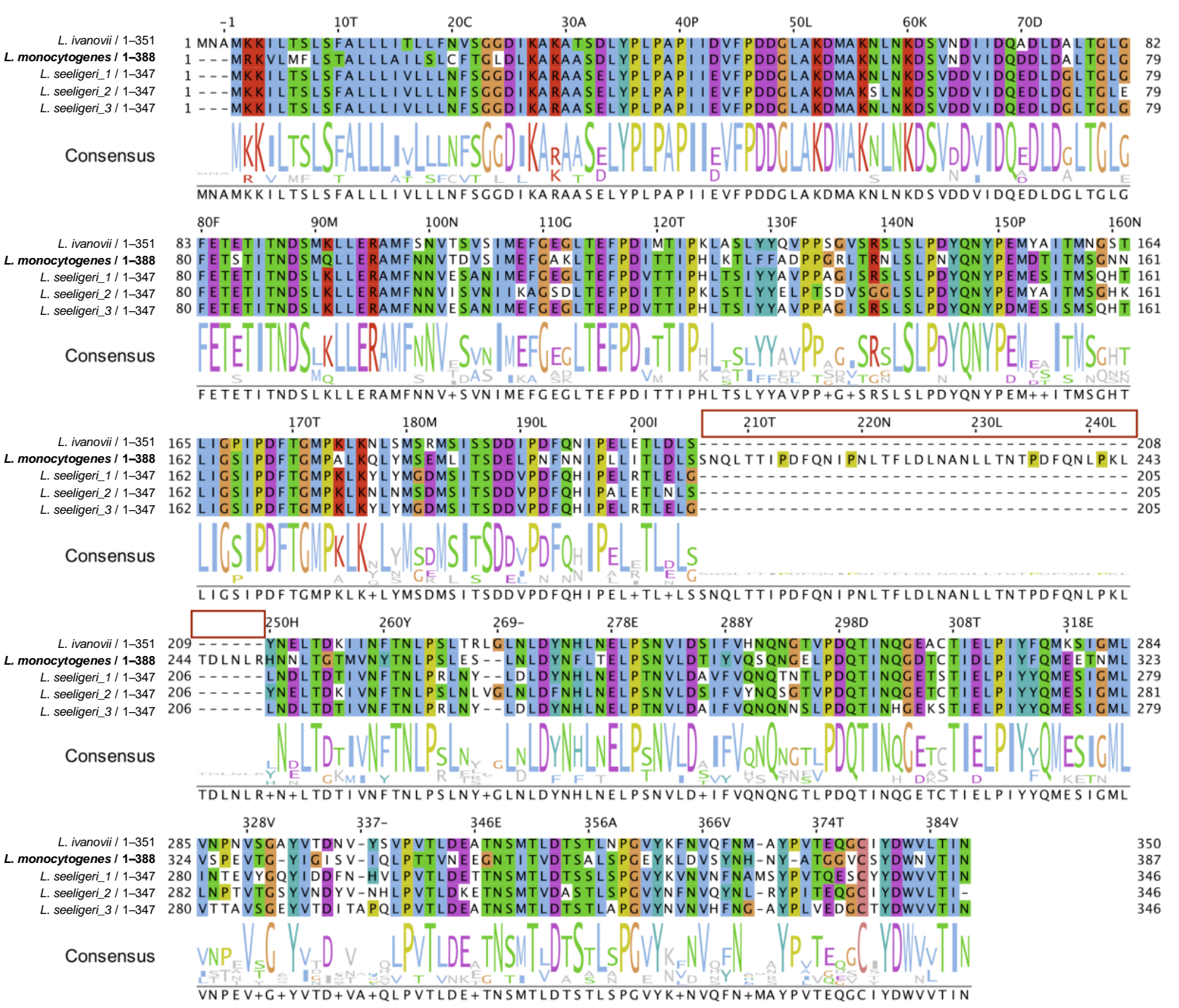




\section{Supplementary Figures}

447 Figure S1: Phobius and Gene3D Domain Architectures of Identified Internalin P

448 Homologs. Cellular localizations (Phobius) and domain architectures (Gene3D) were

449 determined and visualized using MolEvolvR. Figure legends correspond to different 450 domain predictions.

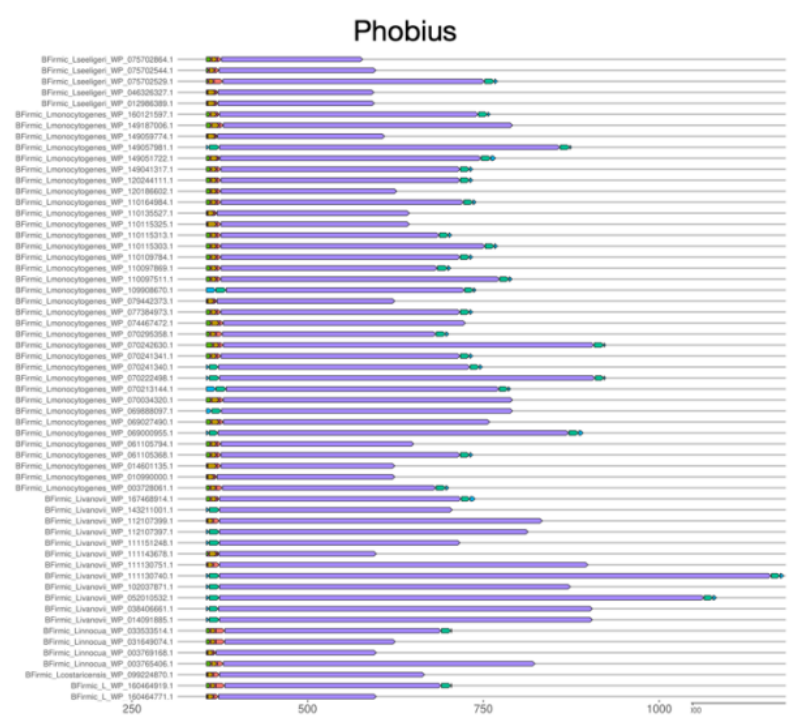

C-serminas rogion of a signal pepside

Hydrophobic region of a signal popicide.

N-terminal region of a signal pepsoce

.

Signal peppide region

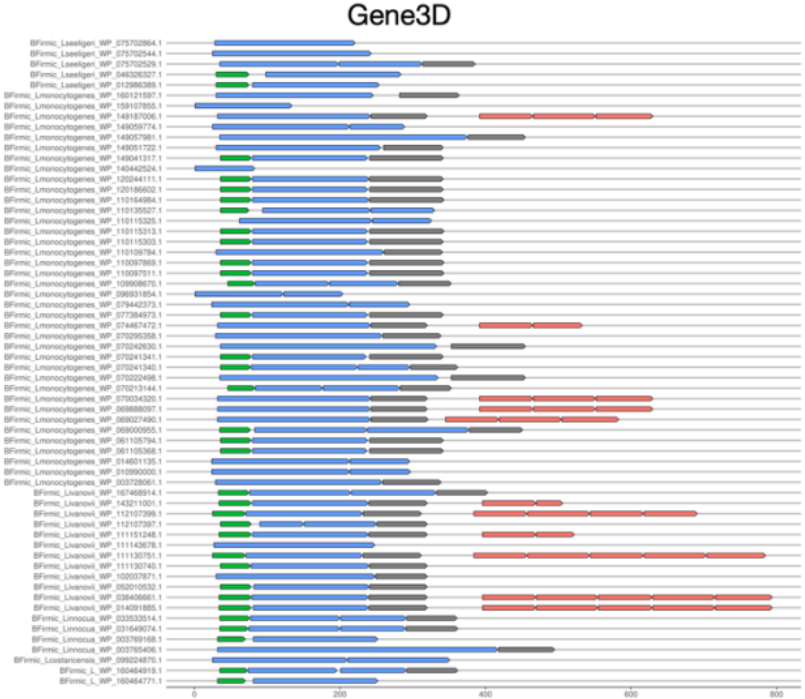

GW domain supertamily

Internalin $\mathrm{N}$-1ermina Cap comain-like

Ribonuchase Inhibitor 
464 Figure S2: Percent Nucleotide Identity of InIP Homolog Genes in $L$. 465 monocytogenes, $L$. ivanovii londoniensis, and $L$. seeligeri. Heatmap representing 466 pairwise percent identities of the nucleotide sequences of Internalin $P$ homologs in $L$. 467 monocytogenes, L. ivanovii londoniensis, and L. seeligeri. Darker and lighter shades of 468 blue represent higher and lower percent identities, respectively.

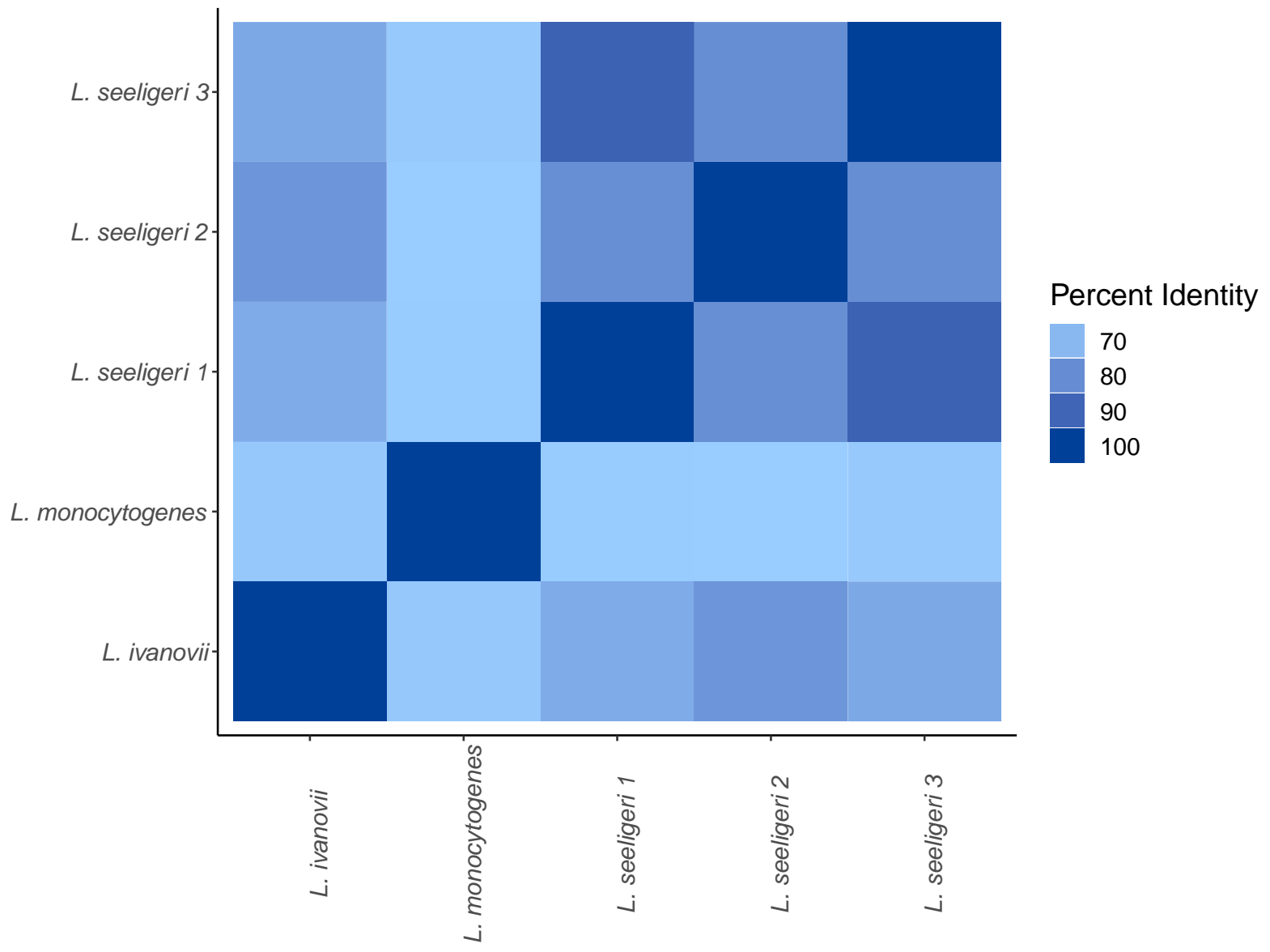




\section{References}

481 1. Goldenberg, R. L., Culhane, J. F., lams, J. D. \& Romero, R. Epidemiology and 482 causes of preterm birth. Lancet 371, 75-84 (2008).

483 2. Lowe, D. E., Robbins, J. R. \& Bakardjiev, A. I. Animal and Human Tissue Models of $484 \quad$ Vertical Listeria monocytogenes Transmission and Implications for Other 485 Pregnancy-Associated Infections. Infect. Immun. 86, (2018).

486 3. Morrison, H. A., Lowe, D., Robbins, J. R. \& Bakardjiev, A. I. In Vivo Virulence 487 Characterization of Pregnancy-Associated Listeria monocytogenes Infections. Infect. Immun. 86, (2018).

489 4. Orsi, R. H. \& Wiedmann, M. Characteristics and distribution of Listeria spp., 490 including Listeria species newly described since 2009. Appl Microbiol Biotechnol 100, 5273-5287 (2016).

492 5. Vazquez-Boland, J. A. et al. Listeria Pathogenesis and Molecular Virulence 493 Determinants. Clinical Microbiology Reviews 14, 584-640 (2001).

494 6. Matereke, L. T. \& Okoh, A. I. Listeria monocytogenes Virulence, Antimicrobial 495 Resistance and Environmental Persistence: A Review. Pathogens 9, 528 (2020).

496 7. Lecuit, M. et al. Targeting and crossing of the human maternofetal barrier by Listeria 497 monocytogenes: Role of internalin interaction with trophoblast E-cadherin.

$498 \quad$ Proceedings of the National Academy of Sciences 101, 6152-6157 (2004).

499 8. Le Monnier, A. et al. ActA Is Required for Crossing of the Fetoplacental Barrier by $500 \quad$ Listeria monocytogenes. Infection and Immunity 75, 950-957 (2007).

501 9. Faralla, C. et al. InIP, a New Virulence Factor with Strong Placental Tropism. Infect. 502 Immun. 84, 3584-3596 (2016). 
503 10. Faralla, C. et al. Listeria monocytogenes InIP interacts with afadin and facilitates

504 basement membrane crossing. PLoS Pathog 14, e1007094 (2018).

505 11. Ireton, K., Mortuza, R., Gyanwali, G. C., Gianfelice, A. \& Hussain, M. Role of

506 internalin proteins in the pathogenesis of Listeria monocytogenes. Mol Microbiol

$507 \quad$ mmi.14836 (2021) doi:10.1111/mmi.14836.

508 12. Kobe, B. The leucine-rich repeat as a protein recognition motif. Current Opinion in

509 Structural Biology 11, 725-732 (2001).

510 13. Hain, T. et al. Pathogenomics of Listeria spp. International Journal of Medical

$511 \quad$ Microbiology 297, 541-557 (2007).

512 14. Glaser, P. et al. Comparative Genomics of Listeria Species. Science 294, 849-852

513 (2001).

514 15. Gouin, E., Mengaud, J. \& Cossart, P. The virulence gene cluster of Listeria

515 monocytogenes is also present in Listeria ivanovii, an animal pathogen, and Listeria

516 seeligeri, a nonpathogenic species. Infect Immun 62, 3550-3553 (1994).

517 16. Burke, J., Chen, S., Sosinski, L., Johnson, J. \& Ravi, J. MolEvolvR: A web-app for

518 characterizing proteins using molecular evolution and phylogeny. In Preparation.

519 17. O'Leary, N. A. et al. Reference sequence (RefSeq) database at NCBI: current

520 status, taxonomic expansion, and functional annotation. Nucleic Acids Res 44,

521 D733-D745 (2016).

522 18. Karp, P. D. et al. The BioCyc collection of microbial genomes and metabolic

523 pathways. Briefings in Bioinformatics 20, 1085-1093 (2019).

524 19. Sievers, F. et al. Fast, scalable generation of high-quality protein multiple sequence

525 alignments using Clustal Omega. Mol Syst Biol 7, 539 (2011). 
526 20. Stothard, P. The Sequence Manipulation Suite: JavaScript programs for analyzing

527 and formatting protein and DNA sequences. Biotechniques 28, 1102-1104 (2000).

528 21. Campanella, J. J., Bitincka, L. \& Smalley, J. MatGAT: an application that generates

529 similarity/identity matrices using protein or DNA sequences. BMC Bioinformatics 4 ,

$530 \quad 29$ (2003).

531 22. R Core Team. R: A Language and Environment for Statistical Computing. (R

$532 \quad$ Foundation for Statistical Computing, 2021).

533 23. Wickham, H. ggplot2: Elegant Graphics for Data Analysis. (Springer International

$534 \quad$ Publishing : Imprint: Springer, 2016). doi:10.1007/978-3-319-24277-4.

535 24. Lassmann, T. \& Sonnhammer, E. L. Kalign - an accurate and fast multiple

536 sequence alignment algorithm. BMC Bioinformatics 6, 298 (2005).

537 25. Waterhouse, A. M., Procter, J. B., Martin, D. M. A., Clamp, M. \& Barton, G. J.

538 Jalview Version 2--a multiple sequence alignment editor and analysis workbench.

539 Bioinformatics 25, 1189-1191 (2009).

540 26. Waterhouse, A. et al. SWISS-MODEL: homology modelling of protein structures and

$541 \quad$ complexes. Nucleic Acids Research 46, W296-W303 (2018).

542 27. Berman, H. M. The Protein Data Bank. Nucleic Acids Research 28, 235-242 (2000).

543 28. Boerlin, P. et al. Listeria ivanovii subsp. Iondoniensis subsp. nov. International

$544 \quad$ Journal of Systematic Bacteriology 42, 69-73 (1992).

545 29. Hupfeld, M. et al. A functional type II-A CRISPR-Cas system from Listeria enables

546 efficient genome editing of large non-integrating bacteriophage. Nucleic Acids

$547 \quad$ Research 46, 6920-6933 (2018). 
bioRxiv preprint doi: https://doi.org/10.1101/2022.01.19.476994; this version posted January 20, 2022. The copyright holder for this preprint (which was not certified by peer review) is the author/funder, who has granted bioRxiv a license to display the preprint in perpetuity. It is made available under aCC-BY-NC-ND 4.0 International license.

548 30. Rocourt, J., Hof, H., Schrettenbrunner, A., Malinverni, R. \& Bille, J. [Acute purulent

$549 \quad$ Listeria seeligeri meningitis in an immunocompetent adult]. Schweiz Med

$550 \quad$ Wochenschr 116, 248-251 (1986). 